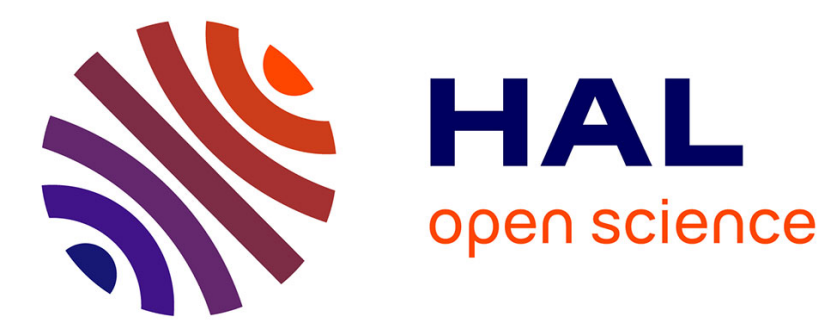

\title{
Jeu et addiction
}

M. Bronnec, B. Rocher, G. Bouju, J.-L. Venisse

\section{To cite this version:}

M. Bronnec, B. Rocher, G. Bouju, J.-L. Venisse. Jeu et addiction. Annales Médico-Psychologiques, Revue Psychiatrique, 2010, 168 (7), pp.509. 10.1016/j.amp.2010.06.004 . hal-00672281

\section{HAL Id: hal-00672281 \\ https://hal.science/hal-00672281}

Submitted on 21 Feb 2012

HAL is a multi-disciplinary open access archive for the deposit and dissemination of scientific research documents, whether they are published or not. The documents may come from teaching and research institutions in France or abroad, or from public or private research centers.
L'archive ouverte pluridisciplinaire HAL, est destinée au dépôt et à la diffusion de documents scientifiques de niveau recherche, publiés ou non, émanant des établissements d'enseignement et de recherche français ou étrangers, des laboratoires publics ou privés. 


\section{Accepted Manuscript}

Title: Jeu et addiction

Authors: M. Bronnec, B. Rocher, G. Bouju, J.-L. Venisse

PII:

S0003-4487(10)00205-2

DOI: doi:10.1016/j.amp.2010.06.004

Reference: AMEPSY 1185

To appear in: $\quad$ Annales Médico-Psychologiques

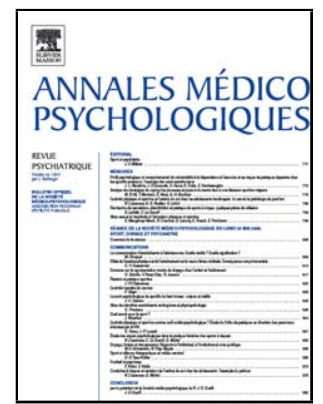

Please cite this article as: Bronnec M, Rocher B, Bouju G, Venisse J-L, Jeu et addiction, Annales medio-psychologiques (2010), doi:10.1016/j.amp.2010.06.004

This is a PDF file of an unedited manuscript that has been accepted for publication. As a service to our customers we are providing this early version of the manuscript. The manuscript will undergo copyediting, typesetting, and review of the resulting proof before it is published in its final form. Please note that during the production process errors may be discovered which could affect the content, and all legal disclaimers that apply to the journal pertain. 


\section{Communication}

Jeu et addiction

\section{Gaming, gambling and addiction}

\section{Bronnec, B. Rocher, G. Bouju, J.-L. Venisse}

Auteur correspondant: J.-L. Venisse, Service d'addictologie, CHU, 9 bis, rue de Bouillé, 44000 Nantes, France

Adresse email : jean-luc.venisse@chu-nantes.fr

\section{Résumé}

L'addiction au jeu concerne principalement les jeux de hasard et d'argent, le jeu pathologique (dont les critères figurent dans le DSM depuis 1980) ayant donné lieu à une littérature scientifique abondante. Les comorbidités psychiatriques et addictives sont fréquentes et les dommages entraînés multiples et sévères. Des distorsions cognitives spécifiques concernant les lois du hasard constituent une cible thérapeutique complémentaire des approches les plus habituelles en addictologie.

Les pratiques excessives de jeux vidéo représentent une problématique émergente justifiant des travaux de recherche afin de mieux cerner leur réalité ainsi que les facteurs de risques impliqués.

Mots clés : Addictions sans drogue ; Addictologie ; Jeu pathologique ; Jeux vidéo

\section{Abstract}

The concept of pathological gambling appeared in the scientific literature around 1980 (DSM-III). Damages associated with pathological gambling are numerous and severe. Psychiatric and addictive comorbidities are frequent. Specific erroneous perceptions and beliefs require cognitive restructuring inside addictologic usual treatment approaches.

Excessive video gaming appears as an emergent phenomenon which justify mores studies to assess its reality and identify risk factors.

Keywords: Addictions without drug; Pathological gambling; Video gaming addictolgy 
Un article de Dupouy et Chatagnon [10], intitulé « Le joueur, esquisse pathologique », paraissait en 1929 dans les Annales Médico-Psychologiques, rapportant l'observation d'un jeune homme de 27 ans hospitalisé quelques mois plus tôt pour un tableau clinique exemplaire de ce qu'on appelle aujourd'hui le jeu pathologique ; il décrit avec une finesse clinique remarquable la situation et la trajectoire de ce joueur de baccarat qui, selon les mots même des auteurs, « joue pour goûter le choc émotif que donne le “coup”, et plus celui-ci est gros, plus celui-là est intense [...] (et) ressemble en cela au toxicomane qui, son éducation faite du toxique, ne désire plus que la sensation, la vibration spéciale que lui procure sa drogue favorite ». Il faudra attendre près de 80 ans pour que cette problématique soit clairement envisagée de façon approfondie en France à l'occasion d'une expertise collective de l’INSERM publiée en 2008, qui est une référence essentielle en la matière [11].

Les pratiques de jeux sont innombrables et ancestrales ; elles font partie de ce qui nous est le plus précieux en termes de plaisir et de développement personnel, dès l'enfance. R. Caillois, dans Les jeux et des hommes [8] définit quatre catégories de jeux, à savoir les jeux de compétition (agon), de hasard (alea), de rôle (mimicry), et de vertige (ilynx). Si on discute la dimension addictive de certaines pratiques dans les trois autres registres que celui de l'alea, le jeu pathologique concerne avant tout les jeux de hasard et d'argent, dans lesquels le joueur vit un temps discontinu, c'est-à-dire où tout est à chaque instant possible.

Les distinctions de plus en plus classiques dans les addictions à des substances psychoactives entre usage simple ou festif, usage à risque, usage nocif ou abus, et dépendance, peuvent tout à fait trouver leur place en matière de pratiques ludiques ; de même que les critères définis par A. Goodman en 1990 [13]. À partir de là, on peut distinguer :

- des joueurs sociaux, pour qui le jeu garde une place limitée, celle d'un loisir (ils sont environ 35 millions en France), dont on peut rapprocher les joueurs professionnels, notamment de cartes, qui en tirent des revenus conséquents, et également le spéculateur boursier ;

- des joueurs problématiques ou excessifs qui sont engagés dans des pratiques de jeux intensives et répétitives et rapportent un certain nombre de dommages au plan financier, personnel, familial et professionnel ;

- enfin, des joueurs pathologiques qui répondent aux critères diagnostiques du jeu pathologique, notamment ceux les plus utilisés du (DSM) tels qui sont rappelés ci-dessous :

\footnotetext{
Critères diagnostiques du Jeu Pathologique

A. Pratique inadaptée, persistante et répétée du jeu, comme en témoignent au moins cinq des manifestations suivantes:

1 Préoccupation par le jeu (p. ex., préoccupation par la remémoration d'expériences de jeu passées ou par la prévision de tentatives prochaines, ou par les moyens de se procurer de l'argent pour jouer)

2 Besoin de jouer avec des sommes d'argent croissantes pour atteindre l'état d'excitation désiré

3 Efforts répétés mais infructueux pour contrôler, réduire ou arrêter la pratique jeu 
La présence de trois ou quatre critères définit le jeu problématique, alors que le diagnostic de jeu pathologique requiert au moins cinq critères.

En l'absence d'études épidémiologiques en population générale concernant la prévalence du jeu à risque et du jeu pathologique en France, il est impossible de préciser la réalité et l'ampleur du phénomène. Dès lors que les taux de prévalence des problèmes de jeu (incluant jeu problématique et pathologique) se situent dans de nombreux pays où des études bien menées ont été réalisées, en particulier en Amérique du Nord, entre 0,5\% et $3 \%$ de la population générale, de façon assez stable dans le temps [9], on peut penser que des taux du même ordre seraient retrouvés en France.

Le développement des jeux de hasard et d'argent sur Internet correspond à un phénomène en pleine expansion qui interroge quant à un risque addictif particulier. Des travaux récents menés à partir des données de la grande étude épidémiologique anglaise de 2007 révèlent une prévalence beaucoup plus élevée du jeu problématique dans le groupe des joueurs en ligne, par rapport à ceux qui ne jouent pas via Internet [15].

\section{Données cliniques}

En marge des définitions académiques du jeu pathologique, pour un repérage clinique simple, un joueur problématique est celui qui joue plus souvent, plus longtemps, et plus qu’il ne peut se le permettre. Chez les joueurs pathologiques, le jeu devient progressivement le centre de l'existence, au détriment de tous les autres investissements affectifs et sociaux, exposant le sujet à de graves conséquences sociales, professionnelles et personnelles. Il est important de souligner que les conséquences du jeu renforcent le comportement et que la connaissance de celles-ci n’empêche pas le joueur de poursuivre cette activité, du fait qu'il entretient l'espoir irrationnel d'un gain important, défiant les lois du hasard.

Les dommages, dont la présence est un des critères majeurs d’addiction, concernent en premier lieu des épisodes dépressifs majeurs, des conduites suicidaires, ainsi que des pertes 
financières (endettement croissant et dans certains cas vente de biens et de domicile, interdit bancaire). Ces dommages participent à la dégradation progressive du climat conjugal et familial autour du joueur pathologique, à l'origine de ruptures et divorces. Dans certains cas, des conduites délictueuses et médico-légales viennent ponctuer ces parcours (vols et malversations financières diverses, en règle sans violence).

Les comorbidités psychiatriques et addictives sont fréquentes. Elles concernent principalement les troubles de l'humeur, les troubles anxieux, ainsi que différentes conduites addictives. Toutes les études ont trouvé une corrélation forte entre jeu pathologique et autres conduites addictives, avec une prévalence du jeu pathologique en tant que conduite actuelle ou passée de l'ordre de 15 \% chez des patients dépendants, quelle que soit la substance, et une prévalence de conduites d'abus ou de dépendance à l'alcool ou à d'autres drogues, actuelles ou passées, de l'ordre de $50 \%$ chez les joueurs pathologiques [19,21].

Les troubles cognitifs retrouvés chez les joueurs pathologiques sont importants et spécifiques. Ladouceur et Gaboury [17] ont été les premiers en 1988 à étudier les pensées dysfonctionnelles des joueurs par la méthode dite de "verbalisation », dans laquelle le joueur exprime à haute voix toutes les pensées qui lui viennent à l'esprit pendant une séquence de jeu. Parmi celles-ci l'illusion de contrôle du hasard (c'est-à-dire une surestimation du degré de contingence entre des actions et leurs résultats) [20], la certitude de gagner quelles que soient les pertes, la certitude que la poursuite du jeu augmente les chances de gagner.

Toutes ces cognitions ne tiennent pas compte des lois qui régissent les jeux de hasard et d'argent, dans lesquelles, par définition, la mise d'argent effectuée par le joueur est irréversible, avec une issue de la séquence de jeu imprévisible et une impossibilité d'exercer sur elle le moindre contrôle. En outre, c'est dans les jeux d'adresse et non de hasard que l'entraînement et la persévération dans le jeu augmentent les compétences et les chances de gagner (le cas des jeux mixtes tel que le Poker Texas H’oldem, très pratiqué actuellement, est un peu particulier, même si, contrairement à ce que pensent beaucoup de joueurs, la part du hasard reste prépondérante).

La trajectoire du joueur en difficulté est traditionnellement décrite à travers une évolution en trois phases successives, avec tout d'abord une phase de gain (et assez souvent la notion d'un gain précoce significatif), puis ensuite une phase de perte et enfin une phase dite de désespoir, lorsque la somme des dommages induits débouche sur une véritable impasse existentielle ; il faut cependant insister sur le constat récent, au vu de suivis de cohortes bien menés, que ces parcours évolutifs sont beaucoup moins linéaires qu’on a pu le penser pendant longtemps, avec de ce fait une absence de stabilité dans le temps du statut de joueur 
pathologique qui est un élément important à prendre en compte dans la compréhension et l’approche thérapeutique de ces troubles [1,25,26].

\section{Facteurs de risque et de vulnérabilité}

Comme dans l'ensemble des addictions, les travaux actuels plaident en faveur d'un déterministe plurifactoriel des conduites de jeu pathologique, au niveau duquel la résonance de facteurs de vulnérabilité et de risque multiples conditionnent, à un moment donné, dans un contexte particulier, la dérive addictive. Certains de ces facteurs sont liés à « l'objet-jeu » luimême (ou encore au contexte de jeu), avec des différences liées au type de jeu en particulier ; d'autres sont plus individuels. Parmi ces derniers, des facteurs psychosociaux ainsi que neurobiologiques et liés aux capacités d’autorégulation, contrôlées mais aussi automatiques, du joueur. On peut également identifier :

- des facteurs liés au genre, avec une nette prédominance masculine des pratiques de jeu, même si cet écart a tendance à se réduire depuis peu $[14,16]$. Le jeu pathologique chez les femmes se caractérise par un début de la pratique plus tardif, un délai de survenue des troubles raccourci, des pratiques de jeu et des motivations à jouer différentes, ainsi que des comorbidités, avant tout anxio-dépressives, alors qu'elles sont souvent addictives chez l'homme [5,23];

- des facteurs liés à l’âge, avec, d'une part, une initiation de ces pratiques souvent durant la période sensible de l'adolescence, la précocité de l'expérimentation étant apparemment un facteur de mauvais pronostic, comme dans beaucoup d'addictions [2,7] et, d’autre part, une vulnérabilité particulière des seniors vis-à-vis du jeu à risque et du jeu pathologique, en lien avec l'isolement et l'inactivité en particulier [29] ;

- des facteurs liés à des antécédents familiaux et personnels, avec la notion d’une prévalence accrue du jeu à risque et pathologique chez les ascendants de joueurs pathologiques, définissant la notion d'agrégation familiale, de même qu’une prévalence accrue chez ces ascendants de conduites addictives, notamment à l'alcool, de traits de personnalité antisociale, et à un moindre niveau d'autres troubles mentaux [4]. Des antécédents personnels d’addictions à des substances psychoactives, et également de troubles précoces du comportement et attentionnels sont de plus en plus évoqués comme des facteurs de vulnérabilité à prendre en compte. En lien, certaines dimensions de personnalité (impulsivité, recherche de sensations, alexithymie...) sont retrouvées chez les joueurs pathologiques. 
La place respective de ces différents facteurs individuels a conduit Blaszczynski [6] à distinguer trois grands types de joueurs pathologiques :

- le premier, constitué de joueurs pathologiques, qu’on pourrait qualifier de « normopathes », c'est-à-dire sans psychopathologie prémorbide, avec des comorbidités dépressives, avant tout conséquences de la conduite de jeu. Ils s’avèrent motivés et compliants aux soins avec de bons résultats à un traitement léger ;

- le deuxième type, qualifié d' «émotionnellement vulnérable », peut utiliser le jeu pour moduler certains états affectifs, de nature anxio-dépressive, en référence à des facteurs de vulnérabilité psychologique, éventuellement reliés à des événements de vie traumatiques, ou encore à des éléments de personnalité borderline, voire des antécédents de problèmes de jeu dans la famille. Ils justifieraient un traitement plus poussé et une exigence d'abstinence totale ;

- le troisième type, enfin, est caractérisé par une dimension impulsive qui se manifeste à travers d'autres troubles du contrôle des impulsions et des conduites. Des éléments de personnalité antisociale, des antécédents personnels de troubles précoces des conduites et notamment de THADA (Trouble hyperactivité avec déficit de l'attention), et souvent des antécédents familiaux de troubles des conduites sont associés, renvoyant à la notion de vulnérabilité neuro-biologique, voire génétique. Souvent peu motivés pour les soins, ces patients nécessitent des programmes de soins spécifiques.

D’un point de vue psychopathologique psychanalytique, le jeu peut être considéré comme un modèle d'addiction sans drogue, que les mots mêmes du joueur illustrent mieux que tout discours. Comment, en effet, mieux décrire la fragilité narcissique impliquée dans beaucoup de processus addictifs, que par l'expression « être à découvert » ? Comment mieux cerner la dimension ordalique de ces pratiques de jeu qu'à travers la formule exemplaire du joueur qui « y retourne pour se refaire »? On a beaucoup insisté depuis Freud et son écrit de 1928 Dostoievski et le Parricide [12], sur les besoins autopunitifs du joueur; dans une perspective névrotique, Freud fait référence à la culpabilité liée au vœu d'inceste oedipien et de parricide. Mais il faut aussi faire une place à la toute-puissance et à la mégalomanie infantile $[3,12]$.

\section{Perspectives thérapeutiques}


L’expérience clinique, comme les données de la littérature, montrent à quel point il est indispensable de recourir avec les joueurs pathologiques aux ressorts thérapeutiques les plus incontournables en addictologie [22].

Peuvent en revanche être considérées comme plus spécifiques les actions directement ciblées au niveau des conséquences propres de la conduite de jeu, ayant valeur de butée structurante dans la réalité : démarche d'interdiction de casino, constitution d'un dossier de surendettement, proposition de mise sous curatelle renforcée, notamment, ainsi que la thérapie cognitive (en individuel ou en groupe) centrée sur les croyances irrationnelles concernant le hasard [18].

\section{Et les jeux vidéo?}

Le problème posé par les jeux vidéo est bien différent de celui des jeux de hasard et d'argent, et la question de l'addiction beaucoup plus discutée, sans validation scientifique internationale en l'état actuel des données. Il est une illustration parmi beaucoup d'autres de la place nouvelle prise par les nouvelles technologies de l'information et de la communication (NTIC) dans nos sociétés et nos vies, qui participent au développement des cyberdépendances, lesquelles se déclinent dans de multiples registres (jeu; relations ; sexe notamment), ayant chacun leur spécificité.

La pratique des jeux vidéo en ligne, et prioritairement des jeux de rôle en réseaux et en univers persistants (MMORPG), les plus concernés par les problèmes d'addictions, a connu un développement exponentiel depuis une vingtaine d'années, de telle sorte qu'une grande majorité d'adolescents et jeunes adultes en ont l'usage, à la différence de leurs parents. C’est ce qui a permis à Tisseron de parler de fracture "générationnelle » pour rendre compte des inquiétudes de parents " obnubilés par quelque chose qui leur échappe », et de préconiser que ceux-ci s’intéressent à la pratique de leurs enfants et les accompagnent dans ce domaine [27].

L’expérience clinique démontre que des interventions de guidance (en individuel ou en groupe) de parents dépassés par la situation les aidant à fixer et tenir des limites (en termes de connexion Internet dans le cas présent), s’avèrent souvent essentielles et suffisantes.

Cependant, dans un certain nombre de cas, et en particulier chez des sujets un peu plus âgés, la pratique s’organise de façon durable et autoentretenue sur un mode très évocateur de dérive addictive. Les critères transversaux de Goodman restent dans ces situations les plus utiles pour différencier les pratiques à risques des conduites d'abus caractérisé, dans lesquels les dommages sont déjà patents (notamment au niveau scolaire, universitaire, ou 
professionnel, mais également parfois du côté des conséquences somatiques d'une insomnie prolongée), et de la dépendance avérée. Le phénomène de centration est alors à son comble avec exclusion de toute autre activité ou investissement et les manifestations de sevrage repérables en cas d'empêchement de pratiquer [24,28].

En l'absence de données épidémiologiques validées, l’importance de ces dérives addictives est difficile à chiffrer. Comme dans d'autres registres addictifs, elles surviennent lorsque des facteurs de vulnérabilité personnelle entrent en résonance avec les effets et impacts de la pratique ludique.

L’expérience clinique révèle que les risques addictifs sont ici également à la mesure des enjeux narcissiques et identificatoires mobilisés chez ceux qui se trouvent les plus démunis à ce niveau, et de ce fait dans une quête sans limite. C’est alors que les sentiments de puissance, mais aussi de dépendance par rapport au groupe (la guilde), de même que l'identification forte à un avatar paré de qualités rêvées, ainsi que la création de fait d'un réseau relationnel virtuel substitutif (chez des sujets présentant une phobie sociale notamment) vont contribuer à une dépendance à la pratique qui s’appuie également sur des sensations corporelles autogénérées répétitivement.

\section{Conclusion}

Le jeu pathologique est une entité reconnue et validée en matière de jeux de hasard et d'argent par une littérature scientifique internationale abondante qui en fait pour certains un modèle d'addiction sans drogue. Les pratiques excessives de jeux vidéo représentent quant à elles une question émergente justifiant des travaux de recherche permettant de mieux cerner leur réalité ainsi que les facteurs de risque impliqués.

\section{Conflit d'intérêt : à compléter par l'auteur}

\section{Références}

[1] Abbot MW, Williams MM, Volberg RA. A prospective study of problem and regular non problem gamblers living in the community. Subt use misuse 2001;39:855-84.

[2] Barnes GM, Welte JW, Hoffman JH, et al. Shared predictors of youthful gambling substance use and delinquency. Psychol Addict Behave 2005;19:165-74.

[3] Bergler E. The Psychology of gambling. USA: International Universities Press; 1957. 
[4] Black DW, Monahan PO, Temkit M, et al. A family study of pathological gambling. Psychiatry Res 2006;141:295-303.

[5] Blanco C, Hasin Ds, Pety N, et al. Sex differences in subclinical and DSM-IV pathological gambling: results from the National Epidemiologic Survey on Alcohol and Related Conditions. Psychol Med 2006;36:943-53.

[6] Blaszczynski A. Pathways to pathological gambling: identifying typologies. Electronic J Gambling Issues 2000;1:1-14.

[7] Burge AN, Pietrzak RH, Molina CA, et al. Age of gambling initiation and severity of gambling and health problems among older adult problem gamblers. Psychiatric Seat 2004;55:1437-9.

[8] Caillois R. Les jeux et des hommes. Paris: Gallimard; 1958, 1991.

[9] Chevallier S, Hamel D, Ladouceur R, Jacques C, Allard D, Sevigny S. Institut National de Santé Publique du Québec, Université de Laval. Comportements de jeu et jeu pathologique selon le type de jeu au Québec en 2002/2004 ; 99 p.

[10] Dupuy R, Chatagnon P. Le joueur esquisse psychologique. Ann Med Psych 1929;2:10212.

[11] Expertise collective de l'INSERM. Jeux de hasard et d'argent - Contextes et addictions. Paris: Les Editions Inserm; 2008.

[12] Freud S. Dostoievski et le parricide. Paris: PUF; 1928.

[13] Goodman A. Addiction: definition and implication. Brit J Addict 1990;85:1403-8.

[14] Grall-Bronnec M, Bouju G, Guillou-Landreat M, Venisse JL. Évaluation sociodémographique, clinique et du parcours de jeu d’un échantillon de joueurs pathologiques français. L’Encéphale, accepté pour publication.

[15] Griffiths M, Wardle H, Orford J, Sproston K, Erens B. Sociodemographic correlates of Internet Gambling: Findings from the 2007 British Gambling Prevalence Survey Cyberpsychology of behaviour 2009;12:199-202.

[16] Ibanez A, Blanco C, Moreyra P, et al. Gender differences in pathological gambling. J Clin Psychiatry 2003;64:295-301.

[17] Ladouceur R, Gaboury A, Dumont D, Rochette P. Gambling: relationship between the frequency of wins and irrational thinking. Journal of Psychology 1988;122:409-14.

[18] Ladouceur R, Sylvain C, Boutin C, Lachance S, Doucet C, Leblond J, Jacques C. Cognitive treatment of pathological gambling. J of Nerv and Ment Disease 2001;189:766-73. [19] Lejoyeux M, Feuche N, Loi S, Solomon J, Ades J. Study of impulse control disorders among alcohol-dependant patients. J Clin Psychiatry 1999;60:302-5. 
[20] Langer EJ. The Illusion of Control. Journal of personality and social psychologic 1975;32:311-28.

[21] Petry NM, Stinson FS, Grant BF. Comorbidity of DSM-IV pathological gambling and other psychiatric disorders; results from the National Epidemiologic Survey on Alcohol and Related Conditions. J Clin Psychiatry 2005;66:564-74.

[22] Petry NM. Disordered Gambling and its treatment. Cognitive and Behavioural Practice 2009;10:1-11.

[23] Potenza MN, Maciejewski PK, Mazure CM. A gender based examination of past year recreational gamblers. J Gambl Study 2006;22:41-64.

[24] Rocher B, Grall-Bronnec M, Vandermersch N, Venisse JL. Les addictions aux jeux vidéo. Archives de Pédiatrie 2008;15:804-5.

[25] Sartor CE, Scherrer JF, Shav KR, Wian H, Volberg F, Eisen SA. Course of pathological gambling symptoms and reliability of the lifetime gambling history measure. Psychiatry Res 2007;152:55-61.

[26] Slutske WS, Jackson KM, Sher KJ. The natural history of problem gambling from age 18 to 29. J Abnorm Psychol 2003;112:263-74.

[27] Tisseron S. Qui a peur des jeux vidéo ? Paris: Albin Michel; 2008.

[28] Valleur M. L’addiction aux jeux vidéo, une dépendance émergente. Enfance Psy 2006. p.125-33.

[29] Vander Blit J, Dodge Hh, Pandav R, et al. Gambling participation and social support among older adults: a longitudinal community study. J Gambl Stud 2004;20:373-89. 